Pluto does or does not have an atmosphere, but methane frost on its surface was discovered in 1976 by Cruikshank, Pilcher and Morrison.

Whyte's The Planet Pluto is one of two very timely recent books about this unique world. Both can be recommended highly. The second book is W. G. Hoyt's Planets $X$ and Pluto (University of Arizona Press), a well-documented, readable account of the discovery and subsequent studies of the planet, based largely on Lowell Observatory records.

Whyte's book is a succinct, thorough summary of our growing knowledge of Pluto. Results are given more or less chronologically without critical comment. This type of presentation has its advantages, but there are occasions where one wishes the author had allowed himself to be more critical. Obvious examples involve Kiladze's numerology, Brady's planet, certain very noisy spectra over-interpreted in fantastic fashions and pedantic models of the planet's interior calculated before the mass or size of Pluto were known.

There are only occasional typos and the odd trivial error, and few important references are omitted. Most notably, no mention is made of such important papers as those of Seidlemann et al. (Publs astr. Soc. Pacif. 84, 858) and Goldreich and Ward (Publs astr. Soc. Pacif. 84, 737) which appeared in 1972 and demonstrated that Brady's huge transNeptunian planet is an impossibility. The references are up to date until July 1979 , and thus do not include some important new results such as the speckle determination of the radius of Pluto by Arnold and his colleagues.

In spite of its high price, the book is recommended heartily to anyone interested in Pluto. It is hoped that it will make a contribution to a much-needed reevaluation of Pluto's importance to our understanding of the Solar System. Perhaps more astronomers will see fit to observe the planet with the techniques now available while it is near perihelion during the next two decades. Pluto has emerged as a unique body in our Solar System and as such is worthy of closer study, and especially of direct exploration by spacecraft. Opportunities exist to fly-by Jupiter - and have another look at Io - and arrive at Pluto before the end of the century.

$J$. Veverka is at the Laboratory for Planetary Studies, Cornell University, New York.

\section{End of a chapter in population genetics}

\begin{tabular}{l}
\hline Alan Robertson \\
\hline Mathematical Population Genetics. \\
Biomathematics Vol. 9. By W.J. Ewens. \\
Pp.322. (Springer-Verlag: 1980.) DM 59, \\
$\$ 33.10$.
\end{tabular}

SINCE the rediscovery of Mendelian segregation, the combinatorial properties of the phenomenon have been fascinating to the mathematician (as indeed they were to Mendel himself). What Ewens calls the "golden age" of mathematical population genetics was dominated by Fisher, Haldane and Wright, the first two with professional training and the latter an inspired amateur. For a time, the theory was far ahead of the relevant evidence but the emergence of new experimental techniques (gel electrophoresis, amino-acid sequencing of proteins and, more recently, DNA sequencing) has not only provided a large body of data, though mostly concerned with protein evolution, but has also stimulated new conceptual approaches, such as the misnamed 'non-Darwinian evolution', and a great deal of sophisticated mathernatics. For instance, our view of the basis of mutation at individual loci is quite altered - we think no longer of 'forward' and 'back' mutation but of an infinite progression which almost never retraces its path and which has led to an 'infinite allele' theory of some elegance.

Many recent theoretical papers seem to have been written for mathematicians rather than biologists. Ewens does an excellent job of presenting the different contributions, many from himself or his students. Aimed at the graduate or research student, two threads are neatly interwoven throughout. Having first presented the theoretical approach to a topic, he ends with his own view of its present biological status. The book deals mainly with the statistics of genes in populations and less than 15 pages are concerned with quantitative genetics. Its value to the reader obviously depends on the latter's mathematical level. I have certainly found it useful and stimulating an indication not only of my mathematics but that I share the author's prejudices.

Perhaps, too, this volume marks the end of a chapter - of a theory of evolution in terms of the substitution of single mutations. Papers dealing with the mathematical properties of systems of tandemly repeated loci have already appeared (though none are referred to here), and current issues of Cell and PNAS are full of discussions of transposable sequences, a new kind of genetic polymorphism, arising from a novel source of variation.

Alan Robertson is Professor at the Institute of Genetics, University of Edinburgh.

\section{The potential of peat}

\section{Hamish Anderson}

Peat: Industrial Chemistry and Technology. By Charles H. Fuchsman. Pp.279. (Academic: 1980.) \$28, £15.80.

AT A time when many countries, faced with ever-declining supplies and resources of basic raw materials, are currently reassessing the potential of alternative sources of energy and of feedstocks for the chemical industry, the appearance of $\mathrm{Dr}$ Fuchsman's book is particularly welcome and opportune. Indeed, this publication constitutes the first real attempt in the English language to collect and collate information on the chemistry and technology of an industry which, until now, has centred largely in eastern and northern Europe. Based on data judiciously selected from over 450 references, including many from Soviet publications not readily accessible to Western scientists, the author presents a most readable and concise review of the nature of organic components in peat and of the alternative technologies available for converting these components into commercially useful and value-added products.

Following a general introduction on the nature and distribution of peat resources and the history of peat utilization for industrial purposes, Chapter 2 presents a somewhat superficial account of the classification and chemical characterization of different bog and peat types, as a result of which the complicated and often ambiguous terminology and nomenclature may trouble the non-specialist. It is also surprising that sapropel, a material now widely used in the USSR, is considered to be of little importance to chemical technology.

The extraction of peat with organic solvents is dealt with in considerable detail in Chapters 3-5, which help to clarify a murky area of the peat research effort. The chemistry and utilization of peat carbohydrates and hydrolysates of peat polysaccharides are comprehensively covered in Chapters 6-8, followed by an account of the hydrolytic production of furfural, lactic and glycolic acids and other sugar byproducts. A further use of these hydrolysates is the indirect production of proteins and fats by the culture of yeasts - a process which has a long and honourable

The second edition of the Glossary of Geology, edited by Robert L. Bates and Julia A. Jackson, has been published by the American Geological Institute, Falls Church, Vermont. The updated Glossary includes 36,000 terms, compared to 33,000 in the 1972 edition, and is available from the AGI, price $\$ 60$. 